$0+2 \nu_{\mathrm{e}}{ }^{\prime} \leftarrow 0$ band taken at a field of $4.68 \mathrm{kV} / \mathrm{cm}$. The upper lines are obtained with the electric vector of exciting light polarized perpendicular to the electric field and the lower ones parallel. For the relatively weak fields employed, the splittings are small, but distinguishable. The results show clearly that the $P$ - and $R$-branch lines are split in parallel polarization while the $Q$-branch lines are split in perpendicular polarization. ${ }^{6}$ This qualitative observation definitely characterizes the transition as magnetic dipole in nature.

Optical Stark spectra have been used to determine dipole moments of electronically excited states..$^{3,4}$ Since the splittings in the present spectra are small, relatively

- It should be noted that the $Q$-branch lines are weaker than the $P$ - and $R$-branch lines. Thus, the assignment of $Q$-branch lines from field-free spectra is not as definite as the strong $P$ - and $R$-branch lines. In the present work the main weight of evidence for the magnetic-dipole nature of the transition comes from the observed Stark splittings of $P$ - and $R$-branch lines. In all cases, the observed splittings of $Q$-branch lines is in accord with their assignment calculated with previously determined constants. We wish to thank the referee for pointing this out. low accuracy can be expected. The value obtained for the dipole moment of the ${ }^{1} A_{2}$ state from measurements of the splittings indicated in Table III is $1.5 \pm 0.3 \mathrm{D}$, which is consistent with the more accurate value of $1.56 \pm 0.07 \mathrm{D}$ determined from splittings measured in the electric-dipole, vibronically allowed $0+\nu_{2}{ }^{\prime}+\nu_{6}{ }^{\prime} \leftarrow 0$ band.,4 It is expected that the variation of dipole moment with vibrational state is less than the present experimental uncertainty of $0.3 \mathrm{D}$.

It appears to us that the present technique is a definitive method for determining the origin of spectral intensity in molecules showing first order Stark effects in their spectra. The general applicability of this straightforward method is probably limited to the spectra of molecular systems with well-characterized rotational structure.

\section{ACKNOWLEDGMENT}

The authors are indebted to Dr. G. Herzberg for suggesting the present experiment.

\title{
Relativistic Effects in Chemical Bonding: The $\mathrm{H}_{2}+$ Molecule
}

\author{
P. I. Pavlik AND S. M. Blinder \\ Chemistry Department, University of Michigan, Ann Arbor, Michigan
}

(Received 8 September 1966)

\begin{abstract}
The behavior of the electron in the ground state of the hydrogen molecular ion is examined using the Dirac theory. Since the resulting set of equations is not readily separable, the variational theorem is applied to obtain approximate eigenfunctions for the ground state. Trial variational functions having the same symmetry properties as the exact solution can be conceptualized as a superposition of functions closely resembling nonrelativistic molecular orbitals. The relativistic correction to the electronic energy is approximately $-7 \times 10^{-6}$ a.u.
\end{abstract}

$\mathbf{T}$ THE methods of nonrelativistic quantum mechanics have been extensively applied to the hydrogen molecule-ion. Within the Born-Oppenheimer approximation, both the exact solution (separable in prolate spheroidal coordinates) and various approximate solutions to the Schrödinger equation for electronic motion have been obtained. The corresponding problem in relativistic quantum mechanics does not appear to have been treated. ${ }^{1}$ The relativistic motion of an electron in the electrostatic field of two protons is governed by the Dirac equation with Hamiltonian operator (in atomic units),

$$
\mathfrak{C}=\mathbf{c} \boldsymbol{\alpha} \cdot \mathbf{p}+\beta c^{2}-\left(\boldsymbol{r}_{\boldsymbol{a}}\right)^{-1}-\left(\boldsymbol{r}_{b}\right)^{-1} .
$$

Unlike the Schrödinger equation, the Dirac equation is not readily separable for the two-center field. We

1 The relativistic two-electron problem has been treated by K. S. Viswanathan, Proc. Indian Acad. Sci. A50, 1 (1959). therefore apply the variational principle to obtain approximate solutions for the relativistic ground state.

For an exact solution to the Dirac equation,

$$
\Psi=\left(\begin{array}{l}
\psi_{1} \\
\psi_{2} \\
\psi_{3} \\
\psi_{4}
\end{array}\right),
$$

the small components $\psi_{3}, \psi_{4}$ of the spinor are related to the large components $\psi_{1}, \psi_{2}$ by $^{2}$

$$
\left(\begin{array}{l}
\psi_{3} \\
\psi_{4}
\end{array}\right)=(2 m c)^{-1} k(\mathbf{r}) \boldsymbol{\sigma} \cdot \mathbf{p}\left(\begin{array}{l}
\psi_{1} \\
\psi_{2}
\end{array}\right)
$$

${ }^{2}$ See, for example, S. M. Blinder, Advan. Quantum Chem. 2, 47 (1965), especially Pp. 53-54. 


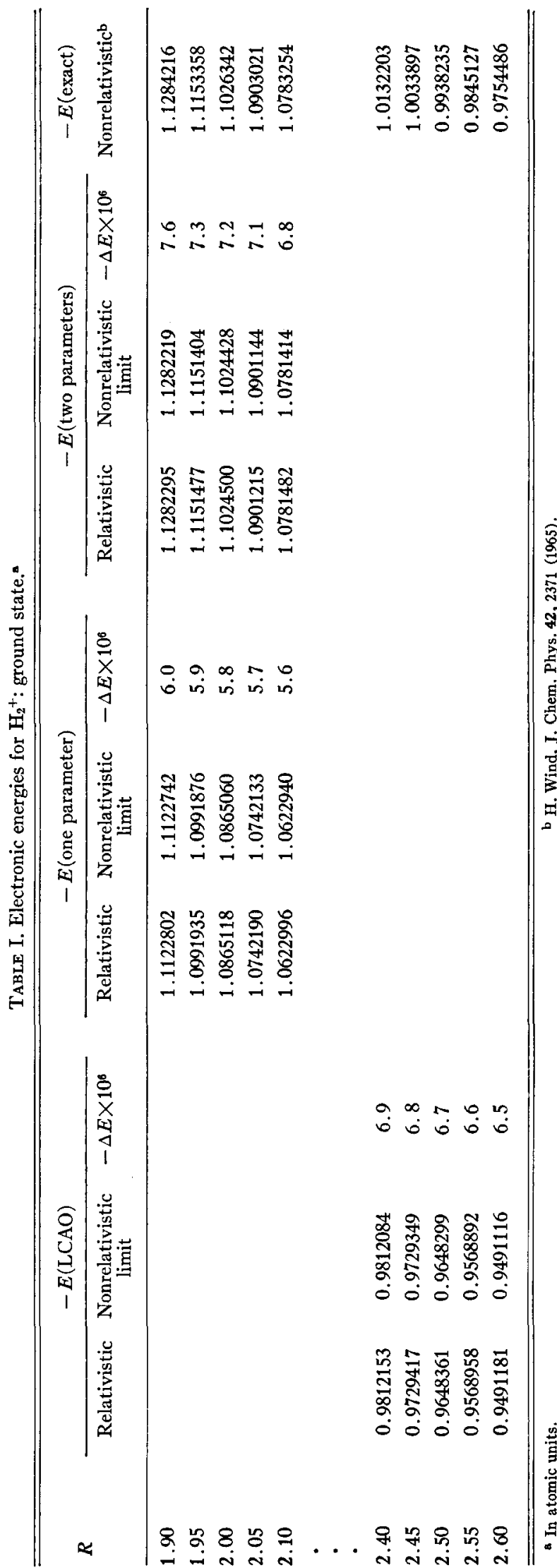

in terms of the function

$$
k(\mathbf{r})=\left(1+\left\{[E+e \phi(\mathbf{r})] / 2 m c^{2}\right\}\right)^{-1},
$$

where $E$ represents the nonrelativistic energy (rest energy subtracted) and $\phi(\mathbf{r})$ is the electrostatic potential.

Accordingly, the expectation value of $E$ can be written as

$$
\begin{aligned}
& E(R)=\left[\Psi,\left(\mathfrak{C}-m c^{2}\right) \Psi\right] \\
& =\sum_{i=1}^{4} \sum_{j=1}^{4}\left\langle\psi_{i}\left|\mathfrak{K}_{i j}-\delta_{i j} m c^{2}\right| \psi_{j}\right\rangle /\langle\Psi \mid \Psi\rangle,
\end{aligned}
$$

where $\mathfrak{H}_{i j}$ is the $i j$ th element of the Dirac Hamiltonian (1). In application of (4), a self-consistency procedure is, strictly speaking, required since the operator depends itself in $E$ through $k(\mathbf{r})$. In practice, however, because of the small magnitude of $E$ relative to $m c^{2}$, even a rough value of $E$ in $k(\mathbf{r})$ suffices. In this article, the expectation value formula (4) is applied to approximate spinor functions which are to be optimized in accordance with the variational principle.

The approximate eigenfunctions were chose as linear combinations of spiniors related to the hydrogen-atom ground state. The spinor representing the relativistic ground state of a hydrogenlike atom with $j=\frac{1}{2}$ and $m_{j}=+\frac{1}{2}$ (spin up) is of the form ${ }^{3}$ :

$$
\Psi=\mathrm{const}\left[\begin{array}{c}
e^{-Z r_{r}} \gamma^{-1} \\
0 \\
i[(1-\gamma) /(1+\gamma)]^{1 / 2} e^{-Z_{r}} \gamma^{\gamma-1} \cos \theta \\
i[(1-\gamma) /(1+\gamma)]^{1 / 2} e^{-z_{r}} \gamma^{\gamma-1} \sin \theta e^{i \phi}
\end{array}\right],
$$

provided that the polar axis is chosen coincident with the spin direction. In (5), $\gamma \equiv\left(1-\alpha^{2} Z^{2}\right)^{1 / 2}$, where $Z$ is the nuclear charge and $\alpha$ is the fine-structure constant $(1 / c$ in atomic units). The first component of (5) corresponds to a $1 s$ nonrelativistic atomic orbital (NRAO) with spin up, apart from the weak singularity at the origin. The third and fourth components have the form, respectively, of a $p_{0}$ NRAO with spin up and a $p_{1}$ NRAO with spin down.

As approximations to the relativistic ground-state molecular orbital for $\mathrm{H}_{2}{ }^{+}$, the following forms for the first component have been considered:

$$
\begin{aligned}
& \psi_{1}=N\left[\exp \left(-r_{a}\right) r_{a}^{\gamma-1}+\exp \left(-r_{b}\right) r_{b}^{\gamma-1}\right], \\
& \psi_{1}=N\left[\exp \left(-\zeta r_{a}\right) r_{a}^{\gamma-1}+\exp \left(-\zeta r_{b}\right) r_{b}^{\gamma-1}\right],
\end{aligned}
$$

and, in terms of prolate spheroidal coordinates $\mu=$ $\left(r_{a}+r_{b}\right) / R, \nu=\left(r_{a}-r_{b}\right) / R$,

$$
\begin{aligned}
\psi_{1}=N & \left\{\exp \left[-\frac{1}{2} R(\zeta \mu+\xi \nu)\right](\mu+\nu)^{\gamma-1}\right. \\
& \left.+\exp \left[-\frac{1}{2} R(\zeta \mu-\xi \nu)\right](\mu-\nu)^{\gamma-1}\right\} .
\end{aligned}
$$

${ }^{3}$ See, for example, H. A. Bethe and E. E. Salpeter, Quantum Mechanics of One- and Two-Electron Atoms (Academic Press Inc., New York, 1957), Sec. 14. 
TABLE II. Relativistic variational results for equilibrium internuclear separation.

\begin{tabular}{lcccccc}
\multicolumn{1}{c}{ Function } & $R$ (a.u.) & $\zeta$ & $\xi$ & $-V$ (a.u.) & $\begin{array}{c}\text { Nonrelativistic } \\
\text { limit }\end{array}$ \\
\hline LCAO $[\mathrm{Eq}$ (6)] & 2.4928 & $\ldots$ & $\ldots$ & 0.5648377 & 0.5648310 \\
Finkelstein-Horowitz [Eq. (7)] & 2.00329 & 1.2380 & $\ldots$ & 0.5865123 & $0.5865065^{\mathrm{s}}$ \\
Guillemin-Zener [Eq. (8)] & 1.9978 & 1.3544 & 0.9190 & 0.6024502 & $0.6026431^{\mathrm{b}}$ \\
Exact solution & 2.00 & $\ldots$ & $\ldots$ & $\ldots$ & $0.6026342^{\mathrm{c}}$ \\
\hline
\end{tabular}

a Reference 9: $V=0.586507,=1.23803, R=2.00329$.

b Reference 10: $V=0.60244,=1.3539,=0.9191$ at $R=2.0$.

These represent the relativistic analogs of the $\mathrm{LCAO},{ }^{4}$ Finkelstein-Horowitz, ${ }^{5}$ and Guillemin-Zener ${ }^{6}$ functions. Since the Hamiltonian (1) commutes with $j_{z}$, the component of total angular momentum along the internuclear axis, the ground state of $\mathrm{H}_{2}{ }^{+}$is doubly degenerate with $m_{j}= \pm \frac{1}{2}$. We need consider only the spinup component of the doublet. For this state, $\psi_{2}=0$. The small components are then determined by (2).? Using (4) to evaluate the energy expectation value and optimizing with respect to the parameters $\zeta$ and $\xi$ the results summarized in Table I are obtained..$^{8}$ The corresponding nonrelativistic limits, approached as $c \rightarrow \infty$ (or $\alpha \rightarrow 0$ ), or by setting $k(\mathbf{r})=1$, are also given. These agree well with recent recalculations based on the Finkelstein-Horowitz ${ }^{9}$ and GuilleminZener ${ }^{10}$ nonrelativistic wavefunctions.

The relativistic contribution to the electronic energy is found to be approximately $-7 \times 10^{-6}$ a.u. This is of the order of $\alpha^{2} E$, as expected for the first-order rela-

${ }^{4}$ L. Pauling, Chem. Rev. 5, 173 (1928).

${ }^{5}$ B. N. Finkelstein and G. E. Horowitz, Z. Physik 48, 188 (1928).

${ }^{6}$ V. Guillemin and C. Zener, Proc. Natl. Acad. Sci. U.S. 15, 314 (1929)

${ }^{7}$ Choice of $\psi$ in this fashion ensures solutions corresponding to positive energy states for a Dirac particle.

${ }^{8} \mathrm{All}$ integrals were evaluated numerically in double precision using $10 \times 10$ Gaussian quadrature and checked by using a finer grid.

${ }_{9}^{9}$ M. Geller, J. Chem. Phys. 36, 2424 (1962); W. J. Meath and J. O. Hirschfelder, ibid. 39, 1135 (1963).

${ }^{10}$ S. Kim, T. Y. Chang, and J. O. Hirschfelder, J. Chem. Phys. 43, $1092(1965)$.
' H. Wind, J. Chem. Phys. 42, 2371 (1965).

tivistic correction to the energy of an electron in such a field.

The approximate internuclear equilibrium separation and corresponding vibrational potential minimum for $\mathrm{H}_{2}{ }^{+}$can be obtained by minimizing

$$
V(R)=E(R)+R^{-1},
$$

with respect to $R$. These values are given in Table II along with the nonrelativistic limits and the results of the corresponding nonrelativistic calculations.

The spinor representing the relativistic molecular orbital is effectively the symmetric linear combination of two hydrogen-atom spinors. The first component of the former, which gives the overwhelmingly dominant fraction of the binding energy, can be identified with a $1 s \sigma_{\theta}$ bonding nonrelativistic molecular orbital (NRMO). Similarly, the third component can be associated with a $p \sigma_{u}$ antibonding NRMO and the fourth component with a $p \pi_{u}$ NRMO. ${ }^{11}$ Evidently the $\pi$ bonding of the fourth component (plus any relativistic enhancement of the bonding of the first component) outweighs slightly the $\sigma$ antibonding of the third component, thus making the relativistic $\mathrm{H}_{2}{ }^{+}$molecule slightly more stable. The relativistic effects operative in $\mathrm{H}_{2}{ }^{+}$are actually at a minimum, owing to the small nuclear charges; they should be considerably magnified in heavier molecules.

11 These have the same symmetry properties as the corresponding components of the exact solution. 